ASSN:1991-8178
EISSN: $2309-8414$
DOI: $10.22587 /$ ajbas.2017.11.15.2
Journal home page: www.ajbasweb.com

\title{
Osteomyelitis of The Rib: A Case Report
}

\author{
${ }^{1}$ Selvakumar Subbaraman MD, ${ }^{2}$ Senthilkumar Selvaraj, MD \\ ${ }^{1}$ Dr. Selvakumar Subbaraman MD, Senior Consultant Radiologist. DNV DIAGNOSTICS, Dharmapuri, Tamilnadu, India \\ ${ }^{2}$ Dr. Senthilkumar Selvaraj, MD, Consultant Radiologist. DNV DIAGNOSTICS, Dharmapuri, Tamilnadu, India.
}

\author{
Address For Correspondence: \\ Dr. Selvakumar Subbaraman MD, AA102, New no.2, 1st Street, 3rd Main Road, Annanagar, Chennai 600040, Tamilnadu, India. \\ Email: drselva73@gmail.com
}

\section{ARTICLE INFO}

Article history:

Received 12 October 2017

Accepted 22 December 2017

Available online 31 December 2017

Keywords:

Osteomyelitis of the rib, rib, pyogenic.

\begin{abstract}
A B S T RA C T
BACKGROUND: Osteomyelitis is infection of the bone. It is common in the long bones like tibia and vertebrae. It occurs frequently in diabetics, patient on steroid therapy, immunocompromised status and in the post operative scenario. Hematogenous spread is the most common route of spread of infection. OBJECTIVE: To discuss the imaging features of the osteomyelitis of the rib. It is a very rare condition and accounts for less than $1 \%$ of hematogenous osteomyelitis. Early recognition allows early treatment. RESULTS: Osteomyelitis of the rib usually has nonspecific clinical manifestations and should be considered even in healthy children. Early recognition allows early treatment. CONCLUSION: Osteomyelitis of the rib is a rare entity in children and should be suspected even when the child is healthy and there are no discharging sinus. With the advent of CT and MRI, the disease can be accurately identified in the early stages resulting in the initiation of appropriate antibiotic or antitubercular therapy
\end{abstract}

\section{INTRODUCTION}

Osteomyelitis is a common condition in the adults but is rare in the paediatric age group. It occurs usually due to hematogenous spread and affects the metaphysis of long bones such as the femur and tibia (Marta Nascimento, et al., 2012). Osteomyelitis of the rib is extremely rare and accounts for less than $1 \%$ of hematogenous osteomyelitis (Kaplan SL., et al., 2005). Osteomyelitis of the rib is associated with childhood empyema., sickle cell anaemia and infection primarily due to staphylococcus aureus, Mycobacterium tuberculosis and Klebsiella species. Osteomyelitis of the rib are known to occur adjacent to the site of insertion of the intercostal drainage tubes in children with empyema (Osinowo, et al., 1982).We report a rare case of pyogenic osteomyelitis involving the right $6^{\text {th }}$ rib.

\section{Case report:}

A 7 year old male child presented to our center with history of pain on the right side at the mid chest level, more on the lateral and posterior aspect, for the past 9 months. There were 2 episodes of fever during this period which resolved with anitobiotics and antiantipyretics. The fever was not high grade. There was no significant history of trauma. Routine blood investigations revealed a hemoglobin of $12 \mathrm{gm} / \mathrm{dl}$, a total leucocyte count of $11,200 / \mathrm{mm} 3$ with $68 \%$ neutrophils. The chest $\mathrm{X}$ ray revealed thickening of the posterior part of the right $6^{\text {th }}$ rib (Fig.1). Hence CT of the thorax (Fig.2,3 \& 4) was done which showed thickening and scleroses of the right $6^{\text {th }}$ rib, on the posterior and lateral aspect, with adjacent soft tissue density collection of $40-50 \mathrm{HU}$ - suggestive of collection - ? empyema. The cortex of the rib appear eroded. The lung fields were clear.

Open Access Journal

Published BY AENSI Publication

(C) 2017 AENSI Publisher All rights reserved

This work is licensed under the Creative Commons Attribution International License (CC BY).

http://creativecommons.org/licenses/by/4.0/

To Cite This Article: Selvakumar Subbaraman MD, Senthilkumar Selvaraj, MD, Osteomyelitis of The Rib: A Case Report. Aust. J. Basic \& Appl. Sci., 11(15): 8-11, 2017 


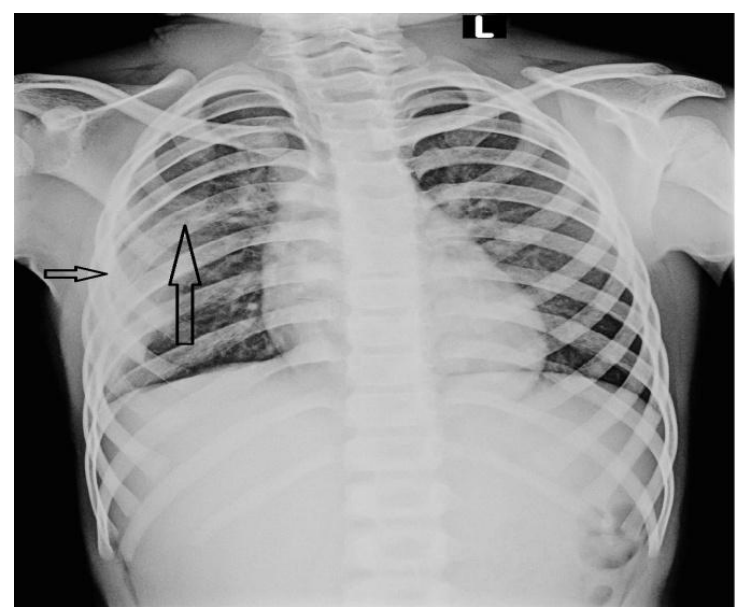

Fig.1: CXR shows thickening of the right $6^{\text {th }}$ rib.

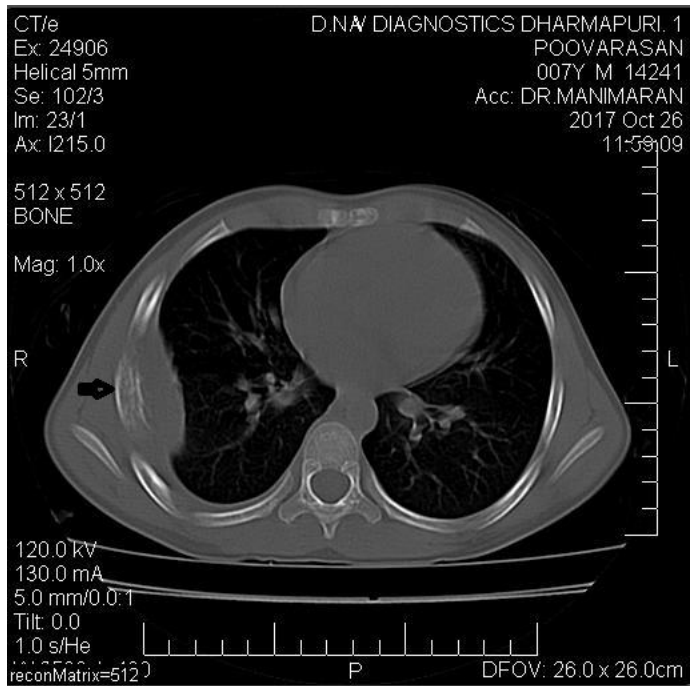

Fig. 2: CT shows thickening of the right $6^{\text {th }}$ rib with cortical erosion.

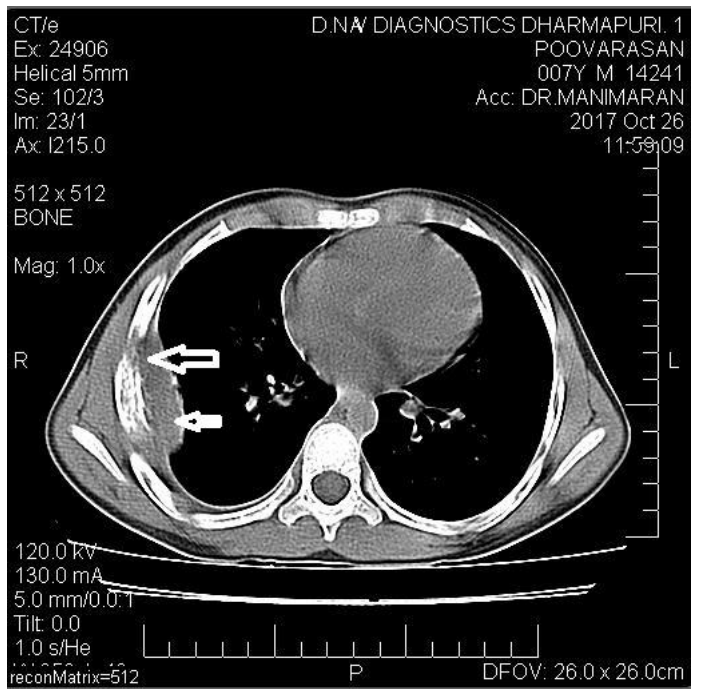

Fig. 3: CT shows cortical thickening and empyema (white arrows). 


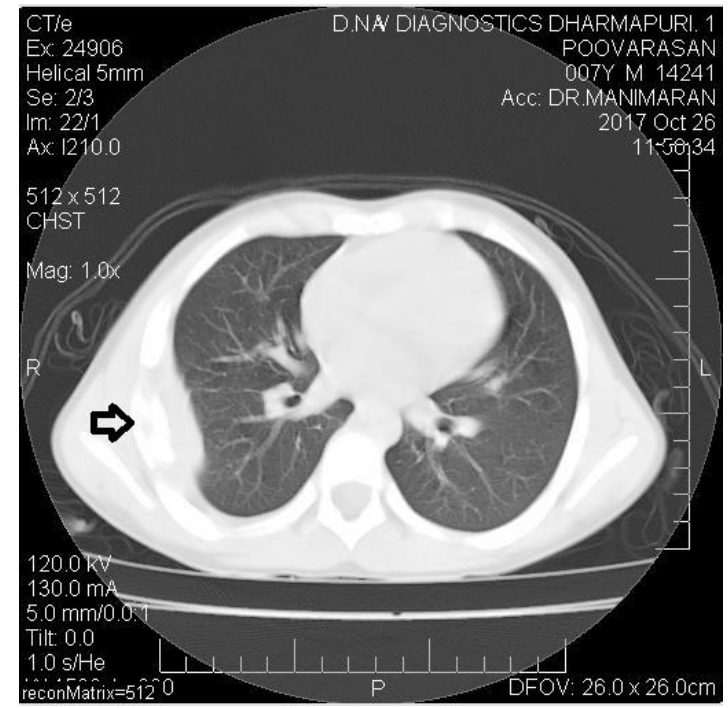

Fig. 4: Lung window shows clear lung fields. The $6^{\text {th }}$ rib is thickened (black arrow).

\section{Discussion:}

Osteomyelitis of the rib is a rare condition and it usually occurs due to (a) penetrating trauma,( b) spread of infection like empyema or pneumonia to the adjacent bone and (c) via hematogenous seeding from a distant focus of infection (Marta Nascimento, et al., 2012; Kaplan SL, et al., 2005). In the case we present, there was no significant history of trauma. We think that the osteomyelitis was due to hematogenous spread. However it cannot be clearly established whether the empyema was a result of the rib infection or vice versa. Osteomyelitis generally involves the metabolically active areas and in regions with the maximum blood supply. The common sites are near the costo-chondral junction anteriorly and the costo-vertebral junction posteriorly. Osteomyelitis involving the rib is generally unifocal. However multifocal lesions have been reported in severely ill newborns (Basa NR, et al., 2004).

The earliest signs and symptoms of the osteomyelitis of the rib are usually nonspecific, which result in delay in diagnosis. The patients generally present with fever, pain and an abscess or sinus that fails to heal (Marta Nascimento, et al., 2012; Osinowo, et al., 1982). The most common infecting pathogen is Staphylococcus aureus. Mycobacterial infection accounts for $7 \%$ cases of osteomyelitis of the rib. Fungal osteomyelitis has been reported in immunocompromised individuals and occurs as a part of disseminated disease (. Conrad DA, et al., 2008). The diagnosis of rib osteomyelitis is relatively fast and accurate with the advent of CT and MRI. The definitive diagnosis of rib osteomyelitis requires culture of the aspirated pus, blood culture or bone biopsy (Kaplan SL; 1982; Conrad DA., et al., 2008). Treatment is usually with intravenous antibiotic or antitubercular therapy (Agrawal V., et al., 2008) depending on the organism grown. Surgical debridement and / or rib resection is done for cases of chronic osteomyelitis (Basa NR, et al., 2004; Basa NR, et al., 2008; Bishara, et al., 2000).

\section{Conclusion:}

Osteomyelitis of the rib is a rare entity in children and should be suspected even when the child is healthy and there are no discharging sinus (Varun Vijay, et al., 2015). Septicaemia and haemoglobinopathies are known to be a major cause osteomyelitis of the rib but these scenario are rarely encountered. Our case did not have any history of blunt chest trauma or insertion of any intercostal drainage tube. In addition to history and a good clinical examination, use of CT-scan, percutaneous needle biopsy, bone scintigram and pathological/mirobiological examination increases the accuracy of the diagnosis (Chang JH, et al., 1999). A biopsy, whether open or CT guided, remains the gold standard for the diagnosis (Jaiswal A, et al., 2006). If the disease can be accurately identified in the early stages resulting in the initiation of appropriate antibiotic or antitubercular therapy (Bishara, et al., 2000; Mohanty D., et al., 2008). Surgical debridement with rib resection is done for chronic osteomyelitis. We had one such case and we take this opportunity to present our rare case report.

\section{REFERENCES}

Agrawal, V., MK., Joshi, BK. Jain, D. Mohanty, 2008.Tuberculotic osteomyelitis of rib--a surgical entity, Interactive Cardiovascular and Thoracic Surgery,7(6): 1028-30. 
Basa, NR., M. Si, F. Ndiforchu, 2004. Staphylococcal rib osteomyelitis in a pediatric patient. Journal of Pediatric Surgery, 39(10): 1576-1577.

Bishara, J., D. Gartman-Israel, M. Weinberger, 2000. Osteomyelitis of the ribs in the antibiotic era, Scandinavian Journal of Infectious Diseases, 32(3): 223-7.

Chang, JH., SK. Kim, WY. Lee, 1999. Diagnostic issues in tuberculosis of the ribs with a review of 12 surgically proven cases, Respirology, 4: 249-253.

Conrad, DA., 2010. Acute hematogenous osteomyelitis. Pediatrics in Review., 31(11): 464-471.

Jaiswal, A., V. Nigam, V. Jain, S. Kapoor, BK. 2006. Dhaon, Multifocal skeletal TB or disseminated bone TB, Tropical Doctor 2006 July, 36: 178-180.

Kaplan, SL., 2005. Osteomyelitis in children. Infectious Diseases Clinics of North America., 19(4): 787-97.

Marta Nascimento, Eliana Oliveira, Sérgia Soares, 2012. Rib osteomyelitis in a pediatric patient: case report and literature review., The Pediatric Infectious Disease Journal. 31(11): 1190-1194.

Mohanty, D., V. Agrawal, 2008. Osteomyelitis of the ribs: a strategy for prompt diagnosis and effective management, Tropical Doctor, 38(4): 239-41.

Osinowo, O., SA. Adebonojo, OA. Adebo, 1982. Childhood empyema in Ibadan, Nigeria, Nigerian Medical Journal, 12: 337-47.

Varun Vijay, Naveen Srivastava, 2015. Chronic Pyogenic Osteomyelitis of the Rib: A Case Report in a Paediatric Patient, Scholarly journal of Applied. Medical.Sciences., 3(3D):1307-1308. 